\title{
Low pericyte coverage of endometrial microvessels in heavy menstrual bleeding correlates with the microvessel expression of VEGF-A
}

\author{
EMIL ANDERSSON ${ }^{1}$, EVA ZETTERBERG $^{2,3}$, INGER VEDIN $^{3}$, KJELL HULTENBY $^{4}$, \\ JAN PALMBLAD $^{3}$ and MIRIAM MINTS ${ }^{1}$
}

\author{
${ }^{1}$ Department of Women's and Children's Health, Karolinska Institutet at Karolinska University Hospital Solna, \\ S-17176 Stockholm; ${ }^{2}$ Departments of Medicine and Hematology, Karolinska Institutet and Karolinska University \\ Hospital Huddinge, S-14186 Stockholm; ${ }^{3}$ Department of Coagulation, Skånes University Hospital, S-21428 Malmö; \\ ${ }^{4}$ Department of Laboratory Medicine, Karolinska Institutet, S-14186 Stockholm, Sweden
}

Received October 15, 2014; Accepted December 10, 2014

DOI: 10.3892/ijmm.2014.2035

\begin{abstract}
A prospective clinical study was carried out to investigate whether endometrial microvessels in patients with idiopathic heavy menstrual bleeding (HMB) of endometrial origin (HMB-E) are fragile due to low pericyte coverage. Idiopathic HMB-E is characterized by large endothelial cell gaps related to the microvascular overexpression of vascular endothelial growth factor (VEGF)-A and VEGF receptors 1-3. A total of 10 women with a normal menstrual cycle and a history of HMB of $<5$ years, and 17 healthy women with a normal menstrual cycle were recruited from the Karolinska University Hospital. Blood samples were obtained for hormone analysis and coagulation tests. Endometrial biopsies were collected in the proliferative or in the secretory phase. Pericyte coverage was assessed using immunohistochemical staining for smooth muscle actin- $\alpha$ (SMA $\alpha$ ) and by image analysis (microvascular density) of endometrial biopsies from 10 patients with HMB-E and 17 healthy ovulating women (control subjects). Previously published data on endothelial cell gap size and the expression of VEGF receptors were used. Although microvascular density did not differ between the patients with HMB-E and the control subjects, the number of SMA $\alpha$-positive microvessels in the proliferative phase was significantly $(\mathrm{P}=0.005)$ lower in the patients with HMB-E than in the control subjects. Moreover, the
\end{abstract}

Correspondence to: Dr Miriam Mints, Department of Women's and Children's Health, Hus 2:0, Karolinska University Hospital Solna, S-17176 Stockholm, Sweden

E-mail: miriam.mints@ki.se

Abbreviations: CI, confidence interval; HMB-E, heavy menstrual bleeding of endometrial origin; HPF, high-power field; MVD, microvascular density; PBAC, pictorial blood loss assessment chart; VEGF, vascular endothelial growth factor

Key words: angiogenesis, endometrium, menorrhagia, gaps, vascular endothelial growth factor, pericytes number of SMA $\alpha$-positive microvessels in the control subjects was significantly fewer in the secretory $(\mathrm{P}=0.04)$ than in the proliferative phase, whereas this number did not differ among the patients with HMB-E regardless of phase. A significant negative correlation was observed between the number of VEGF-A-positive microvessels and microvessels with pericyte coverage $(r=0.8 ; \mathrm{P}=0.04)$. Finally, the endothelial cell layer was significantly thicker in the patients with HMB-E than in the control subjects. Thus, the upregulation of VEGF-A in idiopathic HMB-E is associated with a low pericyte coverage during the proliferative phase of intense angiogenesis, which may confer vessel fragility, possibly leading to excessive blood loss.

\section{Introduction}

Heavy menstrual bleeding (HMB; $>80 \mathrm{ml} / \mathrm{menstrual}$ episode), is common in women of reproductive age, accounting for $>20 \%$ of gynecological outpatient visits. Although HMB is often associated with fibroids and carcinoma, approximately $50 \%$ of HMB cases occur in the absence of recognized uterine pathology. Such idiopathic HMB of endometrial origin (HMB-E) has been estimated to account for 9-14\% of gynecological outpatient visits (1). HMB can lead to iron deficiency and anemia and may require hysterectomy, and its psychological, social and employment consequences for many women have led to its designation as a public health concern (2).

The disturbance of endometrial angiogenesis is one of several mechanisms that have been suggested to play a role in HMB (3). In previous studies, we demonstrated that the agonist-receptor pathway of the vascular endothelial growth factor (VEGF) is upregulated in patients with HMB-E, as they displayed significantly higher numbers of microvessels that expressed VEGF-A, or VEGF receptors-1, -2 and -3 than did the healthy controls (4). Likewise, angiopoietin-1 was upregulated in patients with HMB-E in the secretory phase of the menstrual cycle (5). Moreover, we found that endometrial microvessels possessed a distinct morphology characterized by endothelial cell gaps, and that these gaps (as well as the microvessel perimeters) were larger in patients with HMB-E. 
Endothelial cell gaps highly correlated with the overexpression of VEGF-A and VEGF receptor-1. These findings suggested that microvessel wall cells, such as endothelial cells and possibly pericytes, expressed an aberrant phenotype that may contribute to HMB-E, for instance, by rendering microvessels fragile (6).

An important step in the formation of functional and stable microvessels is the recruitment of pericytes, cells of mesenchymal origin that cover the abluminal surface of endothelial tubes, to form microvessels. Pericytes tightly interact and adhere to endothelial cells through pores in their common basement membrane (7). Pericytes are mostly recruited to endothelial tubes through the expression of platelet-derived growth factor (PDGF), which interacts with its receptor (PDGFR- $\beta$ ) on the pericyte surface. PDGF is secreted mainly by endothelial cells on the tip of the sprouting microvessel, and the amount of PDGF secreted usually determines the extent of pericyte recruitment (8). If the pericytes are insensitive to PDGF due to mutations in the PDGF gene or its receptor, the microvessels become immature and leaky, which leads to widespread hemorrhaging (9). Furthermore, it has been shown that in some cases, pericytes can initiate angiogenesis through the production of VEGF-A, which stimulates endothelial cell proliferation and tube formation (7).

Pericyte coverage can be dense, e.g., in the blood brain barrier, where little and highly regulated transfer of substances occurs, or sparse, e.g., in organs with a high molecule exchange rate across the microvessel wall. Pericytes may also regulate microvessel blood flow, through the contraction of the microvessels (10). One important function of pericytes is to regulate the remodeling of microvessels through pruning of the endothelial network (11).

In the present study, we hypothesized that endometrial microvessels in patients with HMB-E are immature and fragile due to the loss or rearrangement of pericytes. We used previously published data for reported endothelial cell gaps and the expression of VEGF-A, VEGFR1-2 and angiopoietin-1 $(6,12)$.

\section{Subjects and methods}

Study subjects. The data and biological samples used in the present study have been described previously (6). Subjects in this study were recruited from an outpatient clinic for women with HMB. In order to avoid selection bias, patients were enrolled consecutively. Women with a pictorial blood loss assessment chart (PBAC) score $>100$ were considered to have HMB (13). Healthy, ovulating women with a PBAC score $<80$ were considered as the control subjects. All women underwent examination and transvaginal ultrasound, which revealed no pathology in the uterine cavity.

Endometrial biopsies and blood samples for hormones were obtained from 17 control subjects (mean age, 41 years) and from 10 patients (mean age, 42 years) with a normal menstrual cycle and a history of HMB-E of $<5$ years. None of the study subjects smoked, had used drugs (with the exception of tranexamic acid) or hormonal or intrauterine contraception for at least 3 months prior to the biopsy, or had abnormal pre-operative values for blood platelets, activated prothrombin, thromboplastin time, international normalized ratio bleeding time, or were positive for the von Willebrand factor. The uterine cavity of the patients with HMB-E appeared normal as evaluated by hysteroscopy. The date of the last menstruation, the analysis of estradiol and progesterone levels and the histological assessment of endometrial biopsies (which showed no abnormal findings) indicated that 8 patients with HMB-E and 5 control subjects were in the proliferative phase (cycle day 8 ) and 9 patients and 5 control subjects were in the secretory phase of the menstrual cycle (ovulation peak +6 days) at the time of biopsy. The subjects identified the day of luteinizing hormone surge by testing their morning urine.

Ethics statement. All women provided informed consent to participate in the study, which was approved by the Ethics Committee of Karolinska Institutet/Karolinska University Hospital, Sweden.

Immunohistochemical staining for the presence of pericytes. All analyses were performed on the functionalis layer of the endometrium. The processing of endometrial biopsies has been previously described (14). Briefly, 5- $\mu$ m-thick sections of formaldehyde-fixed, paraffin-embedded endothelial tissue were stained with antibodies to the CD34 glycoprotein (Cat. no. ABIN343723; QBEnd/10; BioGenex, San Ramon, CA, USA) alone, or together with smooth muscle actin- $\alpha$ (SMA $\alpha$; M0874; with a FITC conjugated IgG2a; Dako, Glostrup, Denmark), as previously described $(4,5)$. We have previously reported that pericytes in human tissue rarely express PDGFR- $\beta$ and human desmin, but SMA $\alpha$ has been found to reliably identify pericytes in most tissues (6). Thus, the presence of pericytes was determined by SMA $\alpha$ positivity in this study, as previously reported (6).

For secondary antibodies, we used Alexa 488 (I36933) or Alexa 568 (Z-25006; Molecular Probes, Eugene, OR, USA), respectively, as previously described (15). The specificity of these antibodies and their negative controls has been described previously (14).

Quantification of microvessels with pericyte coverage. We used the Leica Q550IW image analysis system, a Leica DM RXA color video camera, and software based on Leica QWin Image Analysis to measure microvessel dimensions following SMA $\alpha$ staining. Ten randomly selected and cross-sectioned CD34stained microvessels, with a clear lumen area (the area of the open space in the microvessel), were captured per slide with a x63 oil immersion objective. Firstly, microvascular density (MVD) was determined, i.e., the number of microvessels per high-power field (HPF). Thereafter, we counted the number of microvessels covered with pericytes. Microvessels with $>50 \%$ of their perimeter covered with pericytes were defined as pericyte-positive microvessels.

Transmission electron microscopy. Small sections of endometrial biopsies were fixed for $30 \mathrm{~min}$ at room temperature, and then for $24 \mathrm{~h}$ at $4{ }^{\circ} \mathrm{C}$ with $2 \%$ glutaraldehyde $+0.5 \%$ paraformaldehyde in $0.1 \mathrm{M}$ sodium cacodylate buffer containing $0.1 \mathrm{M}$ sucrose and $3 \mathrm{mM} \mathrm{CaCl}{ }_{2}, \mathrm{pH} 7.4$ and further processed as previously described (16). To evaluate the thickness of the endothelial cell layer, we performed a volume density measurement, as previously described (17). The total microvessel area was measured on digital images using the software provided with the electron microscope. The total microvessel area (reference area) was calculated using the basement membrane as the 


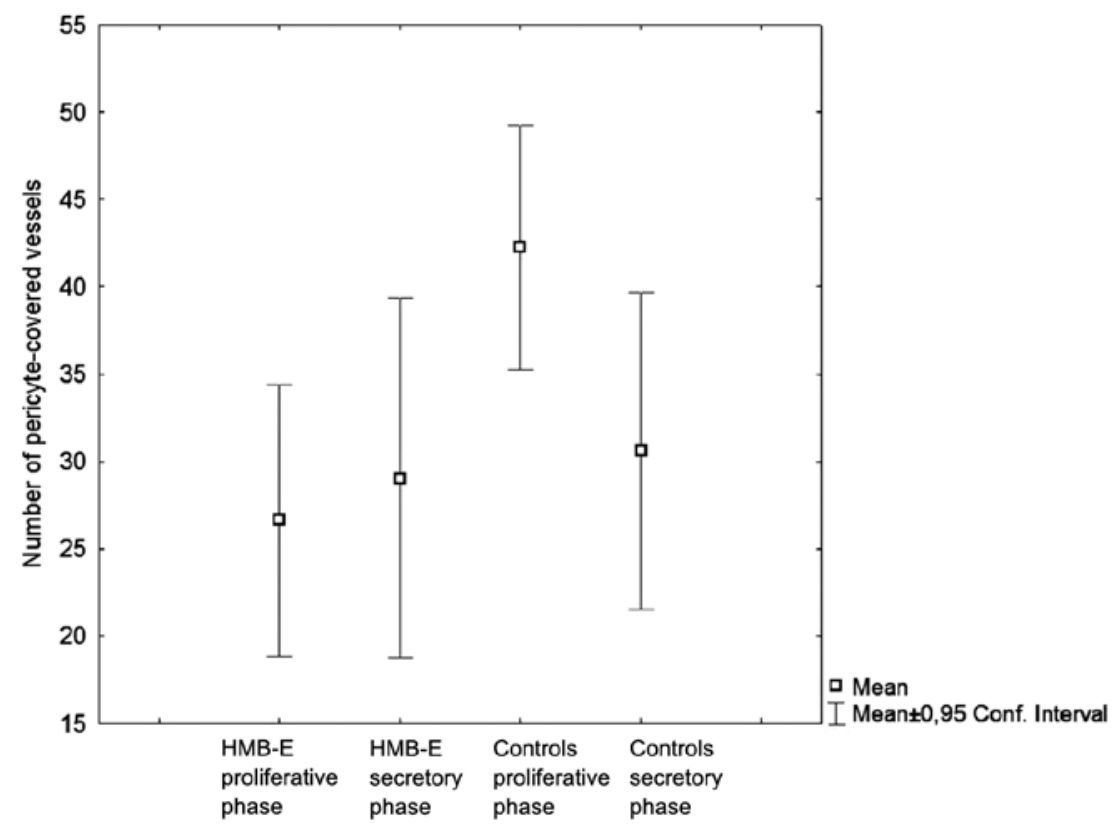

Figure 1. Number of stained smooth muscle actin- $\alpha$ (SMA $\alpha$ )-positive endometrial microvessels in patients and controls by phase of the menstrual cycle. HMB-E, patients with idiopathic heavy menstrual bleeding of endometrial origin. Controls, healthy control subjects. Data are presented as median values and $95 \%$ confidence interval (CI)

outer border. The lumen area was then measured. Dividing the lumen area by the reference area yielded a ratio that represented the volume of endothelial cells; this endothelial area is given as percentage of the reference area.

All endometrial biopsies in the present study were coded and analyzed by two independent observers. Each observer examined the slides on least on two different occasions. Differences in opinion between the observers were resolved by simple discussion and microscopic evaluation. We used previously published data for MVD, endothelial cell gap sizes and the number of microvessels expressing VEGF-A, VEGF receptors 1-3 and angiopoietin-1 $(4,5)$.

Statistical analysis. Data are presented as median values and $95 \%$ confidence intervals (CIs). The Kruskal-Wallis, Mann-Whitney, and Spearman tests were performed with the Statistica software package. A P-value of $<0.05$ was considered to indicate a statistically significant difference.

\section{Results}

MVD, endothelial cell gaps, VEGF-A-positivity and microvessel perimeters. In this study, previously published results $(4-6,14)$ are given, as they were used for correlation analyses. Briefly, MVD did not differ between the patients with HMB-E and the control subjects during the menstrual cycle [median value of 19.5 microvessels/HPF; standard deviation (SD) 6,$0 ; 95 \% \mathrm{CI}$ in patients with HMB-E compared to the median value of 16.0 microvessels/HPF, SD 2, 5; 95\% CI for the control subjects; $\mathrm{P}>0.05]$. Endothelial cell gaps (i.e., discontinuities in CD34, CD31, as well as in von Willebrand factor immunostainings of the endothelial cell layer) have been described in detail in our previous study (6), and were larger in endometrial biopsies from patients with HMB-E than in those from the control subjects. The relative size of endothe- lial cell gaps (i.e., the percentage of the circumference of the microvessels occupied by gaps) in the patients with HMB-E was 1.7 -fold that in the control subjects $(\mathrm{P}=0.000002)$, regardless of the phase of the menstrual cycle. The number of microvessels expressing VEGF-A was significantly $(\mathrm{P}=0.001)$ greater in the patients with HMB-E (median 17; 95\% CI, 16-22) than in the control subjects (median 10;95\% CI, 9-15). The perimeter of endometrial microvessels (measured at the inner, luminal profile of CD34 staining) in the patients with HMB-E in the secretory phase of the menstrual cycle (median, $9.3 \mu \mathrm{m}$; 95\% CI, 6.1-14.9 $\mu \mathrm{m})$ was significantly $(\mathrm{P}=0.0007)$ larger than that for the control subjects in the same phase $(3.6 \mu \mathrm{m} ; 95 \% \mathrm{CI}$, 1.3-7.2 $\mu \mathrm{m})$.

SMA $\alpha$ positivity in endometrial microvessels. The number of SMA $\alpha$-positive microvessels in endometrial biopsies from the patients with HMB-E than in the control subjects (Fig. 1). However, there were no significant differences observed in endometrial biopsies obtained during the secretory phase $(\mathrm{P}=0.87)$.

When we compared the number of SMA $\alpha$-positive microvessels in the control subjects only, we found significantly lower numbers of SMA $\alpha$-positive microvessels in the secretory phase $(\mathrm{P}=0.004)$ compared with the proliferative phase. By contrast, the number of SMA $\alpha$-positive microvessels did not differ among the patients with HMB-E between the two phases. Thus, the control subjects displayed a menstrual cycle-related variation in pericyte coverage, but the patients with HMB-E did not.

Using confocal imaging, we found that the control subjects regularly had CD34-positive microvessels (Fig. 2, control subjects \#1-3). The classic pericyte structure, as disclosed by electron microscopy, is further detailed in Fig. 3 for the control subjects \#1 and \#2. Occasionally, no CD34-positive microvessels were detected, but instead typical pericytes tubes were present (Fig. 2, control subject \#1); in other settings such 

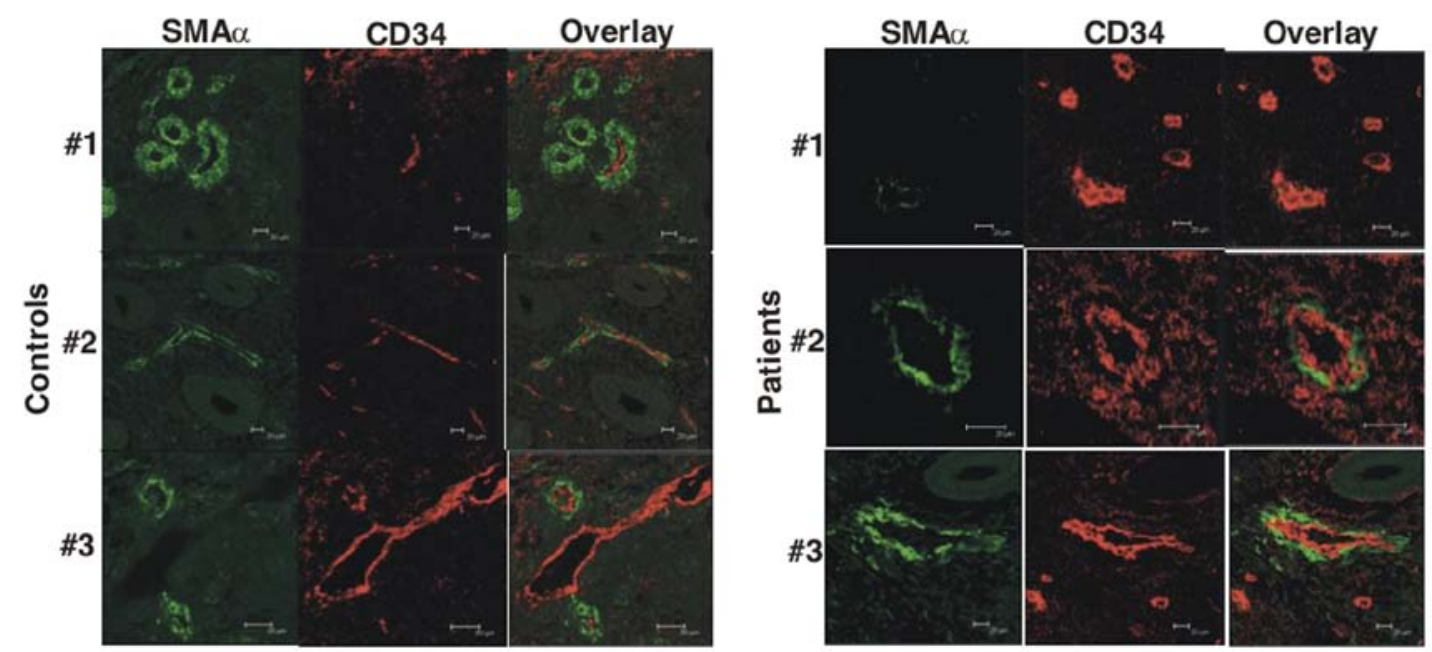

Figure 2. Confocal micrographs of smooth muscle actin- $\alpha$ (SMA $\alpha)$ and CD34 stainings in patients with HMB-E and their overlay in controls. Controls, healthy control subjects \#1, \#2 and \#3. HMB-E, patients with idiopathic heavy menstrual bleeding of endometrial origin \#1, \#2 and \#3. The top row shows microvessels devoid of endothelial cells (in the controls) and microvessels devoid of pericytes (in HMB-E). The two lower rows show the rather slender vessels in the controls in contrast to the coarser vessels in patients with HMB-E.
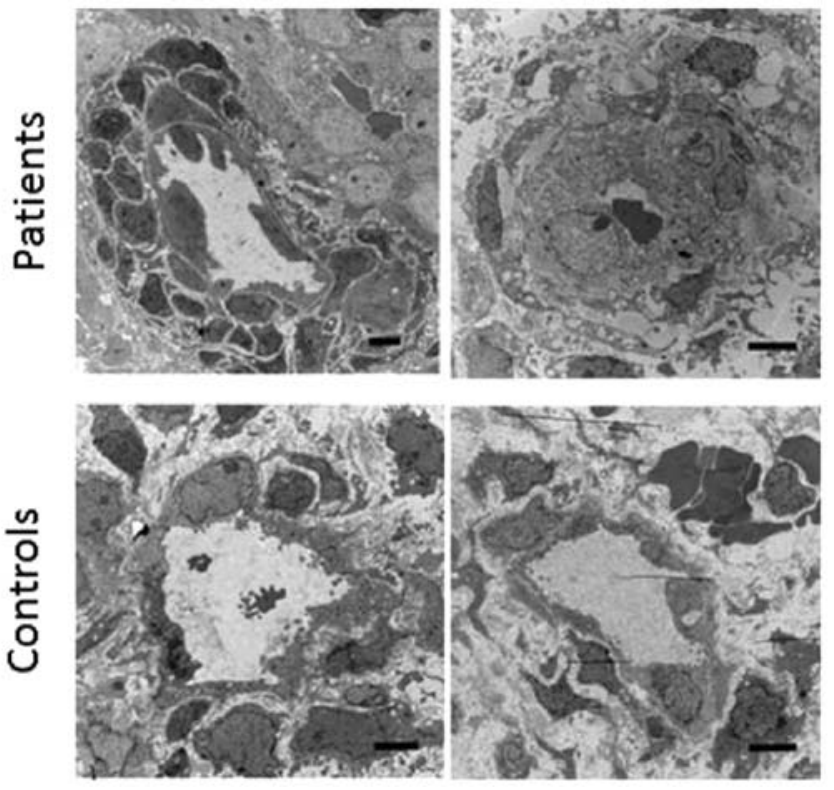

Figure 3. Electron micrographs of microvessels in patients with HMB-E and controls. HMB-E, patients with idiopathic heavy menstrual bleeding of endometrial origin, controls, healthy control subjects. Note the dense accumulation of pericytes in one of the HMB-E (the left panel), as well as the thick endothelial cells in both HMB-E compared to the controls.

structures have been interpreted as remodeling or regressing microvessels, where pericytes remain after endothelial cells have disappeared (18). Only rarely did the control subjects display endothelial tubes without pericytes (Fig. 2, control subject \#3). By contrast, the patients with HMB-E exhibited many endothelial structures without pericytes (Fig. 2, HMB-E patient \#1). Nonetheless, some microvessels showed a combination of endothelial and pericyte structures (Fig. 2, HMB-E patients \#2 and \#3). There was also an impression that microvessels in patients with HMB-E were larger and coarser than those of the control subjects, in that both the CD34 and SMA $\alpha$ stained endothelial cell layers were thicker in the patients with HMB-E than in the control subjects (Fig. 2, HMB-E patient \#2 and \#3). A formal analysis of this finding was performed by means of electron microscopy (see below).

Correlation between pericyte coverage and VEGF-A expression or endothelial cell gaps. Analysis of the data from all patients with HMB-E and the control subjects in the proliferative phase revealed a significant negative correlation between the number of VEGF-A-positive microvessels and the number of microvessels covered with pericytes ( $\mathrm{r}=0.8 ; \mathrm{P}=0.04)$ (Fig. 4). Thus, the higher the VEGF-A expression, the lower the pericyte coverage was.

Analysis of the data from all patients with HMB-E and the control subjects in the proliferative phase revealed no significant correlation between pericyte coverage and the relative size of the endothelial cell gaps, or VEGF receptor-1, -2 or -3 or angiopoietin-1 microvessel expression (data not shown).

Electron microscopy findings. In 2 HMB-E patients and 2 control subjects, 1 HMB-E patient exhibited highly unusual multilayer accumulations of pericytes around the endothelial layer (Fig. 3, left top panel). This was observed in $>90 \%$ of the 7 microvessels examined in this patient. By contrast, the 2 control subjects and the 2nd HMB-E patient displayed traditional pericyte monolayers in all microvessels ( 32 microvessels examined for the 3 subjects) (Fig. 3, right top and bottom panels). With this technique it was not possible to enumerate the pericyte coverage further.

We then analyzed the thickness of the endothelial layer in all microvessels identified in the electron microscopy sections. The patients with HMB-E displayed a significantly higher endothelial cell percentage area compared to the control subjects (median value of $77.4 \%, 95 \%$ CI: 70.4-81.8, $n=17$ microvessels and 53.3\%, 95\% CI: 49.6-58.4, $\mathrm{n}=24$ microvessels, respectively; $\mathrm{P}<0.001)$. There was no evidence of defect contact (e.g., focal adhesion points, caveole) between the pericytes and endothelial cells, mitochondrial morphology abnormalities in the pericytes, or signs of apoptosis or autophagy. 


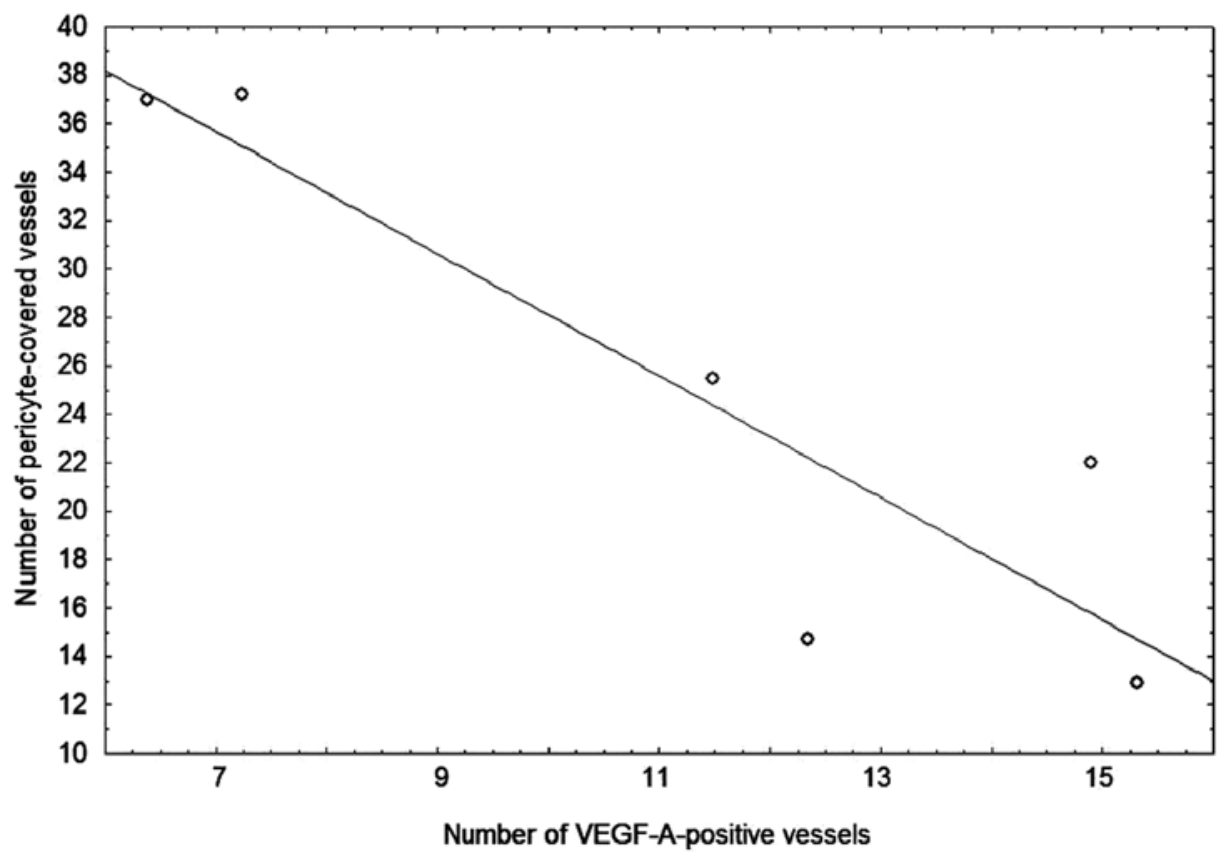

Figure 4. Correlation between the number of VEGF-A-positive microvessels and the number of microvessels with pericyte coverage. VEGF, vascular endothelial growth factor. All patients and control subjects in the proliferative phase for whom values of pericyte coverage and VEGF-A expression were availabe formed the basis for the analysis.

\section{Discussion}

In this study, to the best of our knowledge, we demonstrate for the first time that that the microvessels in the endometrium of women with HMB-E show a dysregulated pericyte coverage: the number of microvessels that were covered with pericytes was significantly lower in the patients with HMB-E during the proliferative phase of the menstrual cycle compared to the control subjects, and the accumulation of pericytes into multilayers occurred in a few women. Moreover, the number of microvessels covered with pericytes negatively correlated with VEGF expression during the proliferative phase. Finally, endothelial linings were thicker in the patients with HMB-E than in the control subjects. Our findings point to a dysregulation of the interaction of endothelial cells and pericytes and suggest that deviant VEGF signaling may be involved in this process.

An important step in the formation of functional and stable microvessels is the recruitment of pericytes. The presence of pericytes is regarded as a sign of vascular maturity (15), while the absence of pericytes is associated with microvessel malformations and microvessel fragility $(19,20)$. Fenestrated microvessels, i.e., microvessels with few pericytes, are more common in the vascular beds of certain organs, whereas other organs have a very tight pericyte surface layer (e.g., the blood brain barrier). Parts of the microvessel where the direct exchange of gas and nutrients takes place are mostly free of pericytes (7), probably as a way to increase the diffusion capacity, whereas the microvessel branchings have high pericyte coverage, believed to be of significance for regulation of blood flow and stress (21).

In the present study, we demonstrated that the number of microvessels covered with pericytes was significantly lower in the patients with HMB-E during the proliferative menstrual phase compared to the control subjects. Moreover, using electron microscopy, we also observed signs of remodeling or regressing microvessels, where pericytes remained after the endothelial cells had disappeared. The lower pericyte coverage in the proliferative phase may lead to the impaired regulation of blood flow, microvessel growth, endothelial cell proliferation and microvessel integrity (10). In all, this would result in poorer microvessel tubes, ultimately leading to a higher probability of microvessel fragility.

We also observed significantly higher pericyte coverage during the proliferative phase than in the secretory phase in the control subjects, while the amount of pericytes during these phases did not differ in the patients with HMB-E. Estrogen receptor- $\beta$ has been found on endothelial cells, which stimulates angiogenesis directly and makes them more sensitive to VEGF-A stimulation (22); therefore the observed differences may depend on the upregulation of VEGF-A by estradiol stimulation. However, the mechanisms through which VEGF-A production and activity is controlled by estrogen and progesterone remains unclear. Estrogen can be either pro- or anti-angiogenic in the endometrium. Studies have indicated that angiogenesis also increases in response to progesterone, and this effect is not inhibited by estrogen $(23,24)$. These same reports demonstrated a significant increase in SMA $\alpha$ positivity in the microvessels of mice treated with progesterone; however, the mechanisms through which these hormones affect the recruitment of pericytes remain unclear. On the other hand, another study illustrated how progestin-only contraceptives reduced the amount of SMA $\alpha$ in the endometrial microvasculature (25), which could indicate an inhibitory role for progesterone on pericytes. The fact that the amount of pericytes did not differ in the patients with HMB-E in our study between the phases of 
the menstrual cycle may indicate a pathological insensitivity to sex hormone stimulation either by the endothelial cells or the pericytes themselves.

Furthermore, we demonstrated that the high local expression of VEGF-A was negatively associated with pericyte coverage. These results are in agreement with the previous demonstration that VEGF-A confers pericyte insensitivity to the recruiting activity of PDGF- $\beta$, ablating pericyte coverage of nascent vascular sprouts, and leading to microvessel destabilization (26).

We did not find a significant correlation between the amount of endothelial cell gaps and the number of microvessels with pericyte coverage. As described in our previous study, endothelial cell gaps were found to be covered with mural cells containing SMA $\alpha$, and these cells were assumed to be pericytes (6). What should be noted is that no leakage of blood was observed near endothelial cell gaps, which may indicate a protective role for pericytes.

In conclusion, our data agree well with, and extend, our previous finding that HMB-E is associated with aberrant angiogenesis. In this study, we demonstrate that an upregulation of the agonist-receptor pathway of VEGF in idiopathic HMB-E affects the recruitment of pericytes, which increases microvessel fragility and possibly leads to excessive blood loss. The evidence of increased fragility of microvessels in the endometrium in patients with HMB-E may provide novel opportunities for investigations and therapeutic interventions.

\section{Acknowledgements}

We thank Annette Hofmann from the Center for Infectious Medicine, Karolinska Institutet, for providing assistance with confocal microscopy. This study was supported by grants from the Swedish Medical Research Council (19X-05991 and 71XS-13135), Karolinska Institutet, the regional agreement on medical training and clinical research (ALF) between Stockholm County Council and the Karolinska Institutet, Huddinge University Hospital, and the Swedish Labour Market Insurance (AFA).

\section{References}

1. Hallberg L, Högdahl AM, Nilsson L and Rybo G: Menstrual blood loss - a population study. Variation at different ages and attempts to define normality. Acta Obstet Gynecol Scand 45: 320-351, 1966.

2. Gath D, Osborn M, Bungay G, Iles S, Day A, Bond A and Passingham C: Psychiatric disorder and gynaecological symptoms in middle aged women: a community survey. $\mathrm{Br}$ Med J (Clin Res Ed) 294: 213-218, 1987.

3. Salamonsen LA, Kovacs GT and Findlay JK: Current concepts of the mechanisms of menstruation. Baillieres Best Pract Res Clin Obstet Gynaecol 13: 161-179, 1999.

4. Mints M, Blomgren B and Palmblad J: Expression of vascular endothelial growth factor receptor-3 in the endometrium in menorrhagia. Int J Mol Med 19: 909-913, 2007.

5. Mints M, Blomgren B and Palmblad J: Expression of angiopoietins 1,2 and their common receptor tie-2 in relation to the size of endothelial lining gaps and expression of VEGF and VEGF receptors in idiopathic menorrhagia. Fertil Steril 94: 701-707, 2010.
6. Mints M, Hultenby K, Zetterberg E, Blomgren B, Falconer C, Rogers R and Palmblad J: Wall discontinuities and increased expression of vascular endothelial growth factor-A and vascular endothelial growth factor receptors 1 and 2 in endometrial blood vessels of women with menorrhagia. Fertil Steril 88: 691-697, 2007.

7. Gerhardt $\mathrm{H}$ and Betsholtz C: Endothelial-pericyte interactions in angiogenesis. Cell Tissue Res 314: 15-23, 2003.

8. Hellström M, Kalén M, Lindahl P, Abramsson A and Betsholtz C: Role of PDGF-B and PDGFR-beta in recruitment of vascular smooth muscle cells and pericytes during embryonic blood vessel formation in the mouse. Development 126: 3047-3055, 1999.

9. Levéen P, Pekny M, Gebre-Medhin S, Swolin B, Larsson E and Betsholtz C: Mice deficient for PDGF B show renal, cardiovascular, and hematological abnormalities. Genes Dev 8: 1875-1887, 1994.

10. Hirschi KK and D'Amore PA: Pericytes in the microvasculature. Cardiovasc Res 32: 687-698, 1996.

11. Hellström M, Gerhardt H, Kalen M, Li X, Eriksson U, Wolburg H and Betsholtz C: Lack of pericytes leads to endothelial hyperplasia and abnormal vascular morphogenesis. J Cell Biol 153: 543-553, 2001.

12. Hewett P, Nijjar S, Shams M, Morgan S, Gupta J and Ahmed A: Down-regulation of angiopoietin-1 expression in menorrhagia. Am J Pathol 160: 773-780, 2002.

13. Higham JM, O'Brien PM and Shaw RW: Assessment of menstrual blood loss using a pictorial chart. Br J Obstet Gynaecol 97: 734-739, 1990.

14. Mints M, Blomgren B, Falconer C, Fianu-Jonasson A and Palmblad J: Microvascular density, vascular endothelial growth factor A, and its receptors in endometrial blood vessels in patients with menorrhagia. Fertil Steril 84: 692-700, 2005.

15. Zetterberg E, Vannucchi AM, Migliaccio AR, Vainchenker W, Tulliez M, Dickie R, Hasselbalch H, Rogers R and Palmblad J: Pericyte coverage of abnormal blood vessels in myelofibrotic bone marrows. Haematologica 92: 597-604, 2007.

16. Förster C1, Mäkela S, Wärri A, Kietz S, Becker D, Hultenby K, Warner $\mathrm{M}$ and Gustafsson JA: Involvement of estrogen receptor beta in terminal differentiation of mammary gland epithelium. Proc Natl Acad Sci USA 99: 15578-15583, 2002.

17. Weibel E: Stereological Methods: Practical methods for biological morphometry. Academic Press, London, 1979.

18. Mancuso MR, Davis R, Norberg SM, O'Brien S, Sennino B, Nakahara T, Yao VJ, Inai T, Brooks P, Freimark B, Shalinsky DR, Hu-Lowe DD and McDonald DM: Rapid vascular regrowth in tumors after reversal of VEGF inhibition. J Clin Invest 116: 2610-2621, 2006.

19. Dulmovits BM and Herman IM: Microvascular remodeling and wound healing: a role for pericytes. Int J Biochem Cell Biol 44: 1800-1812, 2012.

20. Goddard LM and Iruela-Arispe ML: Cellular and molecular regulation of vascular permeability. Thromb Haemost 109: 407-415, 2013

21. Sims DE and Westfall JA: Analysis of relationships between pericytes and gas exchange capillaries in neonatal and mature bovine lungs. Microvasc Res 25: 333-342, 1983.

22. Girling JE and Rogers PA: Recent advances in endometrial angiogenesis research. Angiogenesis 8: 89-99, 2005.

23. Girling JE, Lederman FL, Walter LM and Rogers PA: Progesterone, but not estrogen, stimulates vessel maturation in the mouse endometrium. Endocrinology 148: 5433-5441, 2007.

24. Girling JE and Rogers PA: Regulation of endometrial vascular remodelling: role of the vascular endothelial growth factor family and the angiopoietin-TIE signalling system. Reproduction 138: 883-893, 2009.

25. Rogers PA, Plunkett D and Affandi B: Perivascular smooth muscle alpha-actin is reduced in the endometrium of women with progestin-only contraceptive breakthrough bleeding. Hum Reprod 15 (Suppl 3): S78-S84, 2000.

26. Greenberg JI, Shields DJ, Barillas SG, Acevedo LM, Murphy E, Huang J, Scheppke L, Stockmann C, Johnson RS, Angle N and Cheresh DA: A role for VEGF as a negative regulator of pericyte function and vessel maturation. Nature 456: 809-813, 2008 